\title{
A relativistic orbit model for the LISA mission to be used in LISA TDI simulators
}

\author{
Sophie Pireaux ${ }^{1}$ and Bertrand Chauvineau ${ }^{2}$ \\ ${ }^{1}$ Department 1, Royal Observatory of Belgium, \\ 3 avenue Circulaire, 1180 Brussels, Belgium \\ email: sophie.pireaux@oma.be \\ ${ }^{2}$ ARTEMIS Department, Observatoire de la Côte d'Azur, \\ Avenue de Copernic, Grasse, France \\ email: bertrand.chauvineau@oca.eu
}

\begin{abstract}
The LISA mission is an interferometer, formed by three spacecraft, that aims at the detection of gravitational waves in the $\left[10^{-4}, 10^{-1}\right] \mathrm{Hz}$ frequency band. Present LISA TDI simulators, aimed at validating the novel Time Delay Interferometry method, use a classical Keplerian orbit model at first order in eccentricity in the gravitational field of a spherical nonrotating Sun, without planets. We propose to use the same model but described in the framework of relativistic gravity, and we focus here on quantifying the differences between classical and relativistic orbits for the LISA spacecraft, under the same assumptions.
\end{abstract}

Keywords. gravitation, relativity, methods: analytical, numericical

\section{Introduction}

The Laser Interferometer Space Antenna (LISA) is a joint ESA-NASA mission to be launched in 2018 (at the earliest) [LISA Pre-Phase A Report (1998)]. From the point of view of France, it involves the CNES and the LISAFrance group [LISA-France (2009)]. LISA consists in 3 spacecraft, each following a free-falling test-mass, and of double laserlinks connecting the free-falling test-masses.

The aim of LISA is the detection of gravitational waves in a frequency band complementary to that of Earth based detectors. Indeed, a gravitational wave is a propagating spacetime deformation. As it passes by, a free-falling mass moves like a cork on an oscillating water surface. So, the interferometric armlength between a pair of free-falling test-masses varies. To detect gravitational waves implies to monitor very precisely the interferometric armlength via phase shifts (or fractional frequency fluctuations): a $\Delta L / L$ of the order of $10^{-23}$ with $L=5 \cdot 10^{9} \mathrm{~m}$, the nominal interdistance between LISA spacecraft.

However, a huge challenge faces LISA. Laser frequency and optical bench noises are well above $\left(\sim 10^{-13}\right.$ in fractional frequency units), by orders of magnitude, the gravitational wave threshold $\left(\sim 10^{-21}\right.$ in fractional frequency units). To solve this problem, a new metrology technique, the so-called Time-Delay Interferometry (TDI) was developed [Shaddock et al. (2003)]. It is based on the precise knowledge of photon time transfer $t_{i j}$ between a pair of LISA spacecraft $i$ and $j$. That is the delay taken by a photon to travel the interdistance $L_{i j}$. At lowest order, that is the $L_{i j}$ distance divided by the speed of light. Moreover, the LISA detector is complex. Its sensitivity depends to a large extent on the different noise contributions and on the efficiency of the TDI novel technique. Therefore, and because a laboratory replica of the system is not totally achievable, the performance of LISA (and of TDI in particular) can only be studied with computer simulations of the different processes involved. Such is the aim of the LISACode software [Petiteau et al. (2008)] developed by the LISAFrance group, or of other simulators in 
the USA, namely Synthetic LISA [Vallisneri (2005)] and LISA Simulator [Cornish et al. (2004)]. Among the processes to be implemented in a LISA simulator, the orbit model of the spacecraft, providing positions, velocities and interdistances of spacecraft needed for TDI, is the subject of the present paper.

\section{The three LISA orbit models used}

The orbits of LISA spacecraft have the following characteristics. The three spacecraft should have a drag-free motion at an average interdistance of $L$, forming a triangle that rotates around its center of mass and follows the Earth in its orbit around the Sun at a distance $a=1$ Astronomical Unit.

From the point of view of orbit models, up to now, simulators for TDI LISA [LISACode: Petiteau et al. (2008), Synthetic LISA: Vallisneri (2005) and LISA Simulator: Cornish et al. (2004)] being used by the Mock LISA Data Challenge (MLDC) task force make the following additional simplifying assumptions. Each spacecraft follows perfectly a test mass that is itself perfectly shielded from non-gravitational forces and feels no constraints (for simplicity, one test mass per spacecraft is modeled). As the gravitational field is concerned, solely a spherical non-rotating Sun is considered. Departures from the above assumptions on orbits are presently considered as part of the noise budget in TDI: residual laser frequency and optical bench noises, detector shot noise, ultra-stable oscillator noise, scattered-light noise, laser-beam pointing instability, acceleration noise, inertial mass noise and others (as specified in Table 1 of reference [Petiteau et al. (2008)]).

In the present study, we considered three different orbit models described in the next subsections.

\subsection{Classical orbits}

The first model follows what is used presently in LISA TDI simulators (LISACode, Synthetic LISA, LISA Simulator): classical orbits. Usually, in orbit determination, Newton's second law of motion around a central body, with additional $1 \mathrm{PN}$ relativistic corrections, are numerically integrated. However, for LISA, up to now, neither relativistic corrections nor planetary perturbations to orbits are considered by the MLDC task force [Petiteau et al. (2008), Vallisneri (2005), Cornish et al. (2004) and Arnaud et al. (2007)]. This means Keplerian orbits for each LISA spacecraft. The Keplerian orbits are furthermore developped up to first order in eccentricity in present MLDCs [Arnaud et al. (2007)]. A special angle, $\nu=\frac{\pi}{3}+\frac{5}{8} \frac{L}{2 a}$, is selected for the plane of the LISA triangle with the ecliptic. Indeed, it was shown to minimise LISA natural armlength variations and make it easier to detect gravitational waves. The 3 spacecraft orbits share the same very small eccentricity, $e=\sqrt{1+\frac{4}{\sqrt{3}} \frac{L}{2 a} \cos \nu+\frac{4}{3}\left(\frac{L}{2 a}\right)^{2}}-1 \simeq 0.0096$. They have also the same orbit inclination, $i=\operatorname{arctg}\left(\frac{\frac{L}{2 a} \sin \nu}{\sqrt{3} / 2+\frac{L}{2 a} \cos \nu}\right)$, and orbital period, $T_{c l} \sim 1$ year. But each spacecraft orbit $k=1,2,3$ is out of phase with respect to number 1 by $\vartheta_{k} \equiv-2(k-1) \frac{\pi}{3}$ [Nayak \& Vinet (1999)].

\subsection{Relativistic Motion Integrator (RMI) method}

Since our aim is to estimate relativistic effects in LISA orbits, we used the Relativistic Motion Integrator (RMI) method [Pireaux et al. (2006), Pireaux \& Chauvineau (2008)]. Instead of integrating Newton plus relativistic corrections, RMI integrates numerically straightaway the relativistic equations of motion, i.e. geodesic equations for a given metric, $g_{\beta \gamma}$, and possibly an additional term for non-gravitational forces. Doing so, both 
classical and relativistic gravitational effects (up to the corresponding order of the chosen metric) are taken into account natively.

When applying this RMI method to LISA, following LISA's simplifying assumptions, there is no non-gravitational force term and thus we integrate

$$
\frac{d^{2} x^{l}}{d t^{2}}=\left[-\Gamma_{\beta \gamma}^{l}\left(x^{\mu}\right)+\frac{1}{c} \Gamma_{\beta \gamma}^{0}\left(x^{\mu}\right) \cdot \frac{d x^{l}}{d t}\right] \cdot \frac{d x^{\beta}}{d t} \cdot \frac{d x^{\gamma}}{d t}
$$

where $l=1,2,3, x^{\alpha=0,1,2,3} \equiv(c t, x, y, z), \Gamma_{\beta \gamma}^{l}$ are Christoffel symbols associated with the metric and $c$ is the speed of light in vacuum. We selected the Barycentric Coordinate Reference System metric recommended by the IAU 2000 resolutions; and in order to compare the RMI relativistic orbits for LISA with the standard classical orbits used for the MLDCs, we used it without planets, for a spherical non-rotating Sun (in which case $\Gamma_{\beta \gamma}^{l}$ does not explicitly depend on $t$ ).

\subsection{Relativistic analytical development}

Our third model for LISA orbits is an analytical development up to first order in the eccentricity $e$ of the orbit and up to first order in $G M / c^{2}$, where $G$ is Newton's constant and $M$, the solar mass [Pireaux \& Chauvineau (2008)].

Why a development at first order in eccentricity? Because LISA's eccentricity is small and because TDI and classical orbit models for LISA used by the MLDC task force have been developed using first order in eccentricity approximations [Arnaud et al. (2007): the pseudo-LISA set of conventions].

Hence, the geodesic equations are developed to obtain the purely relativistic radial, $\Delta r \equiv r_{r e l}-r_{c l}$ ( $r e l$ and ${ }_{c l}$ for relativistic and classical orbit models, respectively), and along track, $\Delta l \equiv a \cdot \Delta \theta \equiv a \cdot\left(\theta_{r e l}-\theta_{c l}\right)$, effects at first Post-Newtonian (PN) order as the sum of a zeroth order, ${ }^{[0]}$, and first order, ${ }^{[1]}$, in eccentricity for each satellite $k=1$ to 3 :

$$
\begin{aligned}
& \stackrel{[0]}{\Delta \theta_{k}}=-6 \frac{G M}{a c^{2}}\left\{\begin{array}{l}
+n_{c l} t-\cos \left(n_{c l} t_{k p}\right) \sin \left(n_{c l}\left(t-t_{k p}\right)\right) \\
-\sin \left(n_{c l} t_{k p}\right) \cos \left(n_{c l}\left(t-t_{k p}\right)\right)
\end{array}\right\}
\end{aligned}
$$

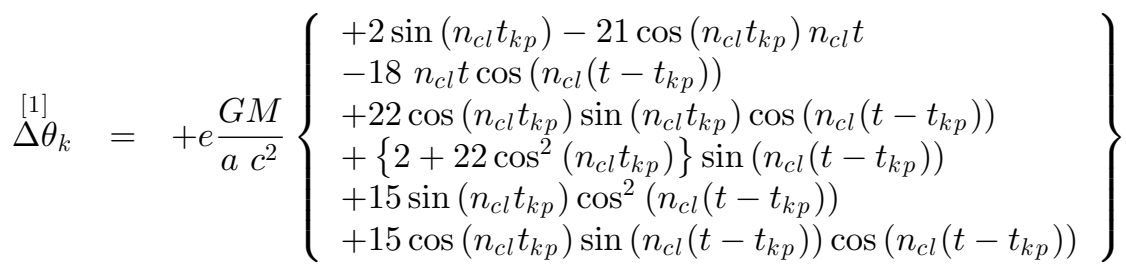

$$
\begin{aligned}
& \stackrel{[0]}{\Delta r_{k}}=+3 \frac{G M}{c^{2}}\left\{\begin{array}{l}
+1-\cos \left(n_{c l} t_{k p}\right) \cos \left(n_{c l}\left(t-t_{k p}\right)\right) \\
+\sin \left(n_{c l} t_{k p}\right) \sin \left(n_{c l}\left(t-t_{k p}\right)\right)
\end{array}\right\}
\end{aligned}
$$

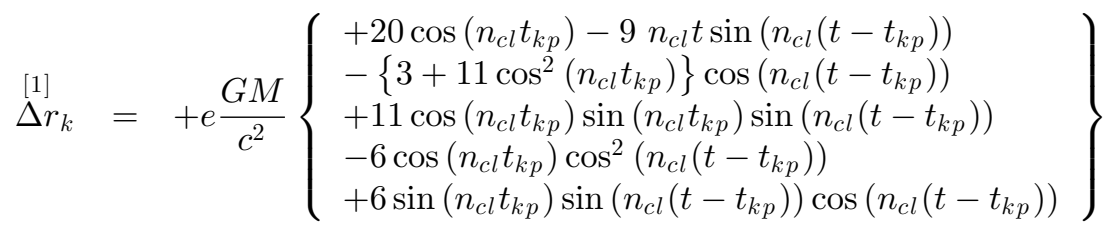

We see that these expressions, in polar coordinates $(x=r \cos \theta, y=r \sin \theta)$, as functions of the derivative of the classical angle, $n_{c l} \equiv d \theta_{c l} / d t$, and of the time of passage of spacecraft $k$ at perihelion, $t_{k p}$, already contain quite a few terms. 

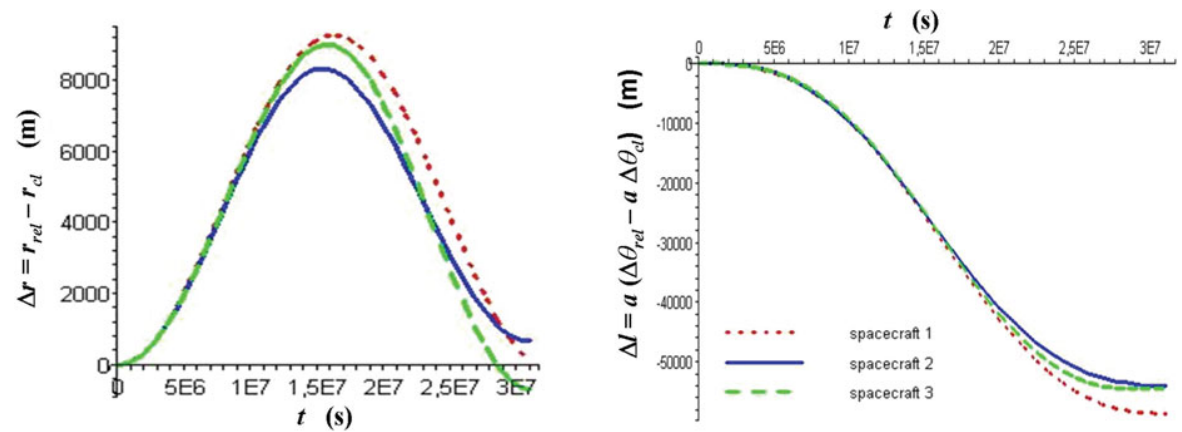

Figure 1. Purely general relativistic effect in [a, left]: radial distance $(\Delta r)$; [b, right]: along track distance $(\Delta l)$.

\section{Orbit model comparison}

We now compare the different orbit models used. First, we check that the spacecraft ephemerides produced by RMI agree with the analytical development at first order in $G M / c^{2}$ and in eccentricity, up to $e^{2} \cdot G M / c^{2}$ terms.

Second, we estimate the purely relativistic effects $(\Delta r$ and $\Delta l)$ in LISA orbits by computing the differences between relativistic spacecraft ephemerides obtained with RMI and classical ones for the same initial coordinate conditions. Figure 1a shows $\Delta r$ for the three LISA spacecraft for one classical period, as a function of the barycentric coordinate time. We see that this radial effect can reach about $9 \mathrm{~km}$.

Figure $1 \mathrm{~b}$ shows $\Delta l$ for one classical period: it can reach about $60 \mathrm{~km}$.

These relativistic effects impact on the satellite interdistance $L_{i j}$ which is crucial in a TDI analysis. We first recall that even classically, the LISA triangle breathes around the nominal interferometric armlength of 5 million $\mathrm{km}$. The armlength flexing amplitude is about 48 000km. Now, relativistic effects add a small contribution to the flexing: up to $3 \mathrm{~km}$ after a period, as is shown in Figure 2. This additional relativistic contribution to the arm flexing is interesting for the TDI method since it impacts on the time taken by photons to travel along those interferometric arms. Indeed, at zeroth order in $1 / c^{2}$, the nominal 5 million $\mathrm{km}$ armlength means $16.7 \mathrm{~s}$ of travel time; the flexing amplitude of $48000 \mathrm{~km}$ means $0.16 \mathrm{~s}$ fluctuation. At half order, the Sagnac and parallax effects amount to $960 \mathrm{~km}$, that is $3 \cdot 10^{-3} \mathrm{~s}$. While at first order, the Shapiro contribution, thanks to the supressing effect of LISA configuration, is less than $30 \mathrm{~m}$, that is $10^{-7} \mathrm{~s}$, as we computed in [Chauvineau et al. (2005)], with the classical orbit model. Now, we have shown that purely relativistic effects in orbits cause a correction up to $3 \mathrm{~km}$ in a period, meaning an effect of several milliseconds in the photon flight time at zeroth order in $1 / c^{2}$.

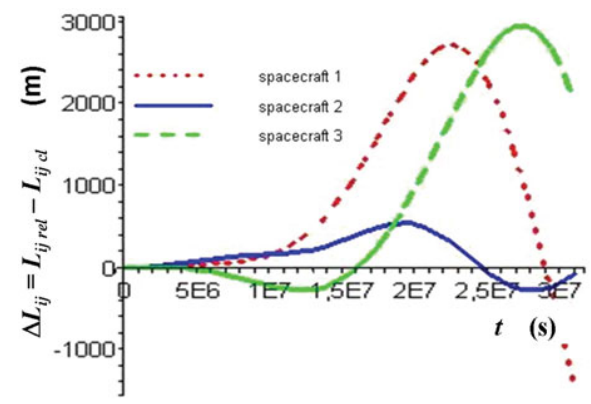

Figure 2. Purely general relativistic effect in armlength. 


\section{Conclusions}

The big relativistic picture in LISA. Time Delay Interferometry (TDI) is a novel technique for spacecraft constellations interconnected by laser links. TDI data-preprocessing [Shaddock et al. (2003)] should help mitigate laser frequency and optical bench noises in interferometry, and bring them down to LISA specifications. TDI ranging [Tinto et al. (2005)] will allow to measure the photon time transfer between two spacecraft. Using an appropriate (with respect to requested precision) laser link model, one can then compute the spacecraft relative interdistances. The LISA mission is a good example to stress that a coherent general relativistic approach is needed. In particular since LISA is a very complex mission and the TDI method must be validated. Hence, the need for LISA TDI simulators. We stress that coherence is needed, in those, between the photon time transfer model [Chauvineau et al. (2005)], the orbit model, coordinates and timescales transformations [Pireaux (2007)]. We studied here the second point.

Relativistic orbit model for LISA TDI simulators. Since, in the simulator named LISACode, as well as in other LISA TDI simulators used by the LISA Mock Data Challenge (LMDC) task force, the orbit model used so far is classical while the laser link is relativistic, we needed to quantify the impact of relativistic effects in LISA orbits on TDI. We have shown that a numerical classical model for LISA orbits in the gravitational field of a non-rotating spherical Sun without planets can be wrong, with respect to the relativistic version of the same model, by as much as about ten kilometers in radial distance during a year and up to about 60 kilometers in along track distance or $3 \mathrm{~km}$ in terms of spacecraft interdistance after a year... with consequences on estimated photon flight times between spacecraft used in TDI.

Note that relativistic orbits for the LISA spacecraft, taking into account planetary and the Moon perturbations, have been obtained through the integration of the EinsteinInfeld-Hoffmann (EIH) equations [Folkner et al. (1997)] and are used for the sake of orbit selection/analysis/formation control studies.

Strength of the Relativistic Motion Integrator (RMI) method. RMI can be used to compute relativistic orbits for different missions (whether barycentric or planetocentric): only the central body parameters and initial conditions, mission parameters in the corresponding RMI modules change. When updating or changing the metric, only the metric module in RMI needs to be updated. No need to recompute additional analytical developments. Indeed, RMI includes any gravitational contribution at the corresponding order of the metric (whether 1PN or higher). RMI has been validated using a 1PN (PostNewtonian) development [Hees \& Pireaux (2009)] and is a coherent native relativistic approach. It should be preferred to "Newton plus relativistic correction" methods, since analytical developments might become cumbersome as the precision of measurements increases and more than $1 \mathrm{PN}$ terms are required.

\section{References}

Arnaud, K. A., Babak, S., Baker, J. G., Benacquista, M. J., Cornish, N. J., Cutler, C., Finn, L. S., Larson, S. L., Linttenberg, T., Porter, E. K., Vallisneri, V., Vecchio, A., \& Vinet, J-Y. (the Mock LISA Challenge Task Force) 2007, arXiv:0701170v4 (gr-qc)

Chauvineau, B., Pireaux, S., Regimbau, T., \& Vinet, J-Y. 2005, Phys. Rev. D, 72, 122003

Cornish, N. J., Rubbo, L. J., \& Poujade, O. 2004, Phys. Rev. D, 69, 082003

Folkner, W. M., Hechler, F., Sweetser, T. H., Vincent, M. A., \& Bender, P. L., 1997, CQG, 14, 1405-1410; and private communication from Dr. Folkner on the 30th July 2009.

Hees, A. \& Pireaux, S. 2009, this proceedings, 144

LISA-France web site at http://www.apc.univ-paris7.fr/LISA-France/analyse.phtml 
LISA Pre-Phase A Report 1998, 2nd Ed., http://www.srl.caltech.edu/lisa/documents/ PrePhaseA.pdf

Nayak, K. R., \& Vinet, J-Y. 2005, CQG, 22, S437-S443

Petiteau, A., Auger, G., Halloin, H., Jeannin, O., Pireaux, S., Plagnol, E., Regimbau, T., \& Vinet, J-Y. 2008, Phys. Rev. D, 77023002

Pireaux, S., Barriot, J-P., \& Rosenblatt, P. 2006, Acta Astronautica, 59, 517-523; gr-qc/06022008

Pireaux, S. 2007, CQG, 24, 2271-2281

Pireaux, S. \& Chauvineau, B. 2008, arXiv:0801.3627v1(gr-qc)

Shaddock, D. A., Tinto, M., Estabrook, F. B., \& Armstrong, J. W. 2003, Phys. Rev. D, 68, 061303(R)

Tinto, M., Vallisneri, M., \& Armstrong, J. W. 2005, Phys. Rev. D, 71, 041101

Vallisneri, M. 2005, Phys. Rev. D, 71, 022001 